\title{
Sizing and Layout Design of an Aeroelastic Wingbox through Nested Optimization
}

\author{
Bret K. Stanford* \\ NASA Langley Research Center, Hampton, VA, 23681 \\ Christine V. Jutte ${ }^{\dagger}$ \\ Craig Technologies, Inc., Cape Canaveral, FL, 32920 \\ Christian A. Coker $\ddagger$ \\ Mississippi State University, Starkville, MS, 39759
}

\begin{abstract}
The goals of this work are to 1) develop an optimization algorithm that can simultaneously handle a large number of sizing variables and topological layout variables for an aeroelastic wingbox optimization problem and 2) utilize this algorithm to ascertain the benefits of curvilinear wingbox components. The algorithm used here is a nested optimization, where the outer level optimizes the rib and skin stiffener layouts with a surrogate-based optimizer, and the inner level sizes all of the components via gradient-based optimization. Two optimizations are performed: one restricted to straight rib and stiffener components only, the other allowing curved members. A moderate $1.18 \%$ structural mass reduction is obtained through the use of curvilinear members.
\end{abstract}

\section{Introduction}

Layout optimization of a wingbox structure, a form of topology optimization, involves deciding upon the best placement of ribs, spars, and stiffeners within a semimonocoque structure. There are numerous examples of layout design in the literature, including Refs. 1 - 6. Some of these papers adhere to conventional orthogonal layouts of ribs, spars, and stiffeners, ${ }^{3}$ others blend the roles of these components through angled and/or curved members, ${ }^{2,5,6}$ and others abandon this convention entirely with a complex network of fulland partial-depth members. ${ }^{1,4}$

Regardless of how the layout design is conducted, sizing design must be included as well, by optimizing the thickness distribution of the various shell components: ribs, spars, stiffeners, and skins (cover panels). The synergies between these two types of design variables (layout/topology and sizing) are strong, particularly in terms of skin buckling metrics. Fewer stiffeners and/or ribs increase the size of the concomitant skin panels, requiring thicker skins to preserve the required buckling and stress performance. Simultaneous optimization of sizing and topology is required to locate the best compromise to minimize weight, but this poses substantial numerical difficulties. Layout optimization typically must be conducted with non-gradientbased optimization (specifically, evolutionary optimization is used for each of the references above). This family of algorithms scales very poorly with the number of design variables, and so if sizing variables are to be included as well, they must be restricted to a small, diluted set of parameters.

It is obviously preferable to work with a large set of sizing variables for detailed component sizing, driven by an algorithm that scales well with large design spaces (gradient-based optimization). In this case, sequential optimization may be considered, where the topology is optimized under a frozen set of sizing parameters, followed by sizing optimization on a frozen wingbox topology, and so on. This is considered in Ref. 2, but sequential optimization cannot, in general, be expected to fully capture the rich couplings

\footnotetext{
*Research Aerospace Engineer, Aeroelasticity Branch, bret.k.stanford@nasa.gov, AIAA Senior Member.

$\dagger$ Research Engineer, Advanced Materials and Processing Branch, christine.v.jutte@nasa.gov.

$\ddagger$ Undergraduate Research Assistant, Department of Aerospace Engineering, cac853@msstate.edu, AIAA Student Member.
} 
between sizing and topology. Furthermore, layout optimization of ribs/spars/stiffeners in isolation (i.e., with a frozen set of sizing variables) will struggle to minimize structural weight while also maintaining constraints on stress, buckling, flutter, etc. ${ }^{7}$

A superior approach, though computationally expensive, is to utilize a nested optimization considered in Refs. 3 and 8. In this approach, the layout/topology design variables are optimized within the outer level via a global optimizer, while the sizing design variables are handled at the inner level with a gradient-based optimizer. Each set of layout design variables is evaluated by freezing the topological details, and then sizing the wingbox to minimize the structural mass of that particular topology, under a series of aeroelastic performance constraints. The final structural mass (objective function) is then returned to the topology optimizer, which then moves on to the next topology under consideration, and the process repeats. The topology optimizer is not explicitly aware that sizing optimization occurs at the nested level, and is also unaware of the existence of the performance constraints (as the sizing optimization is able to satisfy all constraints). Rather than the relatively inefficient evolutionary topology optimization considered in the previously-cited references, a Bayesian/surrogate-based optimizer is used here; the final algorithm resembles recent work conducted in Ref. 9.

\section{Approach}

\section{A. Wing Layout Generation}

All of the work in this paper is conducted on the conceptual Common Research Model (CRM). The $1 g$ outer mold line for the CRM is described in Ref. 10, and a jig shape CRM wingbox, suitable for aeroelastic analysis, was subsequently developed by Kenway et al. ${ }^{11}$ This transonic transport has a wing span of $58.7 \mathrm{~m}$, a mean aerodynamic chord of $7.0 \mathrm{~m}$, a taper ratio of 0.25 , a sweep angle of $35^{\circ}$, and a cruise Mach number of 0.85 . The aluminum wingbox is composed of upper and lower skins, a leading and trailing spar, ribs, and T-shaped stiffeners. The topology optimizer dictates the number and placement of the ribs and skin stiffeners, though the topology of the two spars is fixed, as is the topology of the vertical web stiffeners. The skins, spars, and ribs are modeled with shell finite elements, the skin stiffeners with beam elements, and the vertical web stiffeners are not modeled explicitly, but instead smeared into the shell stiffness properties ${ }^{12}$ of the webs. Rib stiffener pitch is fixed at $15 \mathrm{~cm}$, and spar stiffener pitch at $23 \mathrm{~cm}$.

The baseline, "empty" wingbox, shown on the left of Fig. 1, is composed of the skins, stiffened spars, a close-out rib, and a rib located at the trailing edge break: these topological features are constant. From this starting point, the layout optimizer adds ribs and skin stiffeners. Two topological parameterizations are considered here, the first utilizing only straight members (Table 1) and the second allowing for curved members (Table 2). For Table 1, stiffener rotations are measured relative to the leading edge spar (positive angles swept forward), and stiffener spacing indicates the ratio of the stiffener pitch at the leading edge to that at the trailing edge. The topology optimizer can separately design the rib layout in the inboard and outboard portions of the wing (delineated by the fixed rib at the trailing edge break). Rib spacing in Table 1 is the ratio of the rib pitch at the two ends of each rib design zone, an idea taken from the "linked shape" parameterization of Ref. 2.

Referencing Table 2, curved stiffener paths are generated by 4 control points located from root to tip, where $2 \cdot y / b$ is the relative semispan location. Stiffener rotation is dictated by the 4 design variables, and is assumed to vary in a piecewise linear manner from point to point. Spanwise integration of this piecewise linear plot yields a curvilinear path. Curved rib layouts are designed using a parameterization taken from Ref. 2, where $\eta_{1}$ and $\eta_{2}$ dictate the normalized chordwise location of a control line at the two ends of each rib design zone, and the various $\xi_{i j}$ terms dictate the relative locations of various control points within the design zone. More details of this "linked shape" parameterization can be found in the reference. It should be noted that if $\eta_{1}=\eta_{2}=0.5, \xi_{12} / \xi_{11}=\xi_{22} / \xi_{21}=\xi_{32} / \xi_{31}$, and the stiffener rotations at all 4 control points are set equal to each other, then the straight-member topological parameterization of Table 1 is recovered. It is finally noted that in each parameterization, 4 of the design variables are integers, while the remainder are continuous.

Two sample topologies are shown in Fig. 1, one straight and one curved. In order to generate the beam/shell finite element meshes of the wingbox, each added topological component (ribs and stiffeners) is meshed independently, and then "stitched" to the outer skins and spars via multipoint constraints (MPCs). Specifically, each node of the skin stiffeners, and each node along the perimeter of a rib, is designated a slave node, whose motion is dictated by the shape functions of the skin/spar shell finite element that circumscribes 


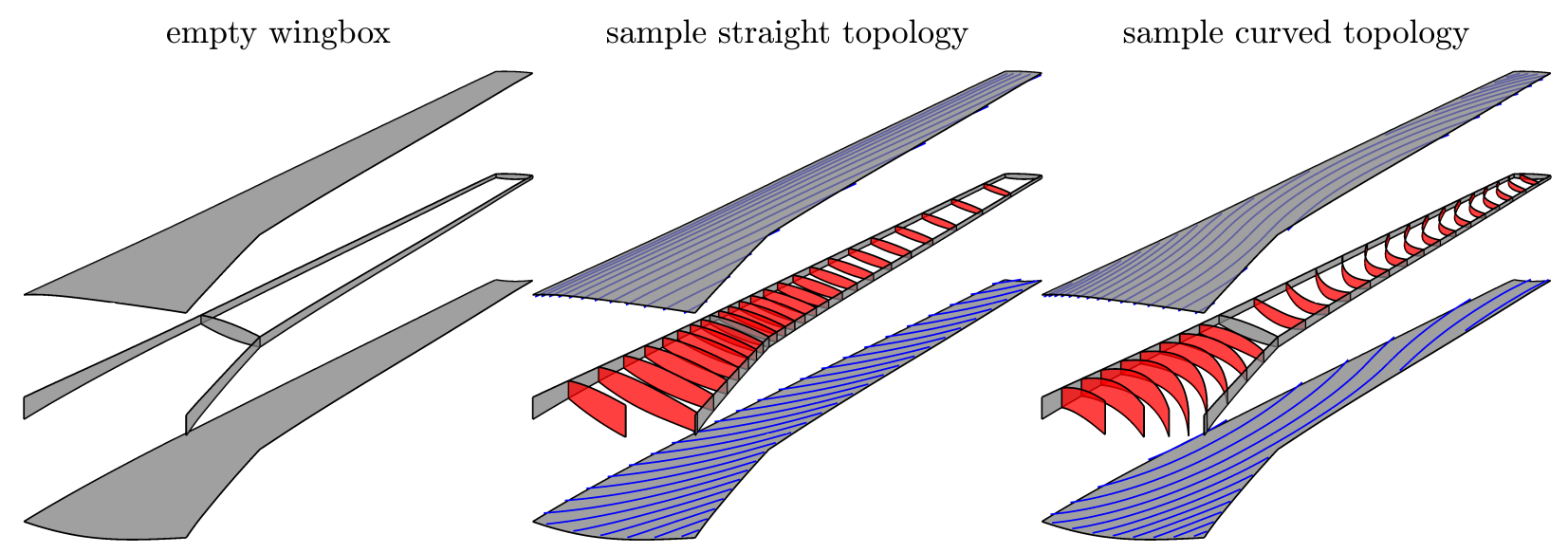

Figure 1. Sample wingbox topologies with straight and curved members.

Table 1. Topological parameterization with straight members only (10 total). "UB" and "LB" are the upper and lower bounds of each variable.

\begin{tabular}{rcc|rcc} 
parameter & LB & UB & parameter & LB & UB \\
\hline \# of upper skin stiffeners & 8 & 20 & \# of inboard ribs & 3 & 14 \\
upper skin stiffener rotation & $-30^{\circ}$ & $30^{\circ}$ & inboard rib spacing & 0.25 & 4.0 \\
upper skin stiffener spacing & 0.25 & 4.0 & \# of outboard ribs & 10 & 26 \\
\# of lower skin stiffeners & 8 & 20 & outboard rib spacing & 0.25 & 4.0 \\
lower skin stiffener rotation & $-30^{\circ}$ & $30^{\circ}$ & & & \\
lower skin stiffener spacing & 0.25 & 4.0 & & &
\end{tabular}

it. This approach is found to be less computationally expensive, and (more importantly) far more robust than a situation where a monolithic mesh is computed in an automated manner (see Ref. 13 for a recent example of this). The topology optimizer will consider a wide range of layouts, each with a large number of components inside the wingbox; any failures of the geometry/meshing engine will leave an unacceptable

Table 2. Topological parameterization with curved members (24 total).

\begin{tabular}{rcc|rcc} 
parameter & LB & UB & parameter & LB & UB \\
\hline \# of upper skin stiffeners & 8 & 20 & \# of inboard ribs & 3 & 14 \\
upper skin stiffener rotation $(2 \cdot y / b=0.0)$ & $-30^{\circ}$ & $30^{\circ}$ & inboard rib $\eta_{1}$ & 0.1 & 0.9 \\
upper skin stiffener rotation $(2 \cdot y / b=0.29)$ & $-30^{\circ}$ & $30^{\circ}$ & inboard rib $\eta_{2}$ & 0.1 & 0.9 \\
upper skin stiffener rotation $(2 \cdot y / b=0.65)$ & $-30^{\circ}$ & $30^{\circ}$ & inboard rib $\xi_{12} / \xi_{11}$ & 0.25 & 4.0 \\
upper skin stiffener rotation $(2 \cdot y / b=1.0)$ & $-30^{\circ}$ & $30^{\circ}$ & inboard rib $\xi_{22} / \xi_{21}$ & 0.25 & 4.0 \\
upper skin stiffener spacing & 0.25 & 4.0 & inboard rib $\xi_{32} / \xi_{31}$ & 0.25 & 4.0 \\
\# of lower skin stiffeners & 8 & 20 & \# of outboard ribs & 10 & 26 \\
lower skin stiffener rotation $(2 \cdot y / b=0.0)$ & $-30^{\circ}$ & $30^{\circ}$ & outboard rib $\eta_{1}$ & 0.1 & 0.9 \\
lower skin stiffener rotation $(2 \cdot y / b=0.29)$ & $-30^{\circ}$ & $30^{\circ}$ & outboard rib $\eta_{2}$ & 0.1 & 0.9 \\
lower skin stiffener rotation $(2 \cdot y / b=0.65)$ & $-30^{\circ}$ & $30^{\circ}$ & outboard rib $\xi_{12} / \xi_{11}$ & 0.25 & 4.0 \\
lower skin stiffener rotation $(2 \cdot y / b=1.0)$ & $-30^{\circ}$ & $30^{\circ}$ & outboard rib $\xi_{22} / \xi_{21}$ & 0.25 & 4.0 \\
lower skin stiffener spacing & 0.25 & 4.0 & inboard rib $\xi_{32} / \xi_{31}$ & 0.25 & 4.0
\end{tabular}


gap in the design space, potentially impeding optimization convergence. The MPC-based meshing algorithm described above never fails, which out-balances the added complexities of handling the geometry constraints.

Having generated and meshed the wingbox topology, the algorithm used here then connects a series of lumped masses to the wing, as shown in the right of Fig. 2. These masses are connected to rib-spar-skin intersection points via interpolation elements, and are meant to represent internal fuel $(62,000 \mathrm{~kg}$ total $)$, the pylon/engine (11,400 kg total), and control devices along the leading and trailing edges $(9,400 \mathrm{~kg}$ total). Unmodeled mass for the half-vehicle is set to $53,000 \mathrm{~kg}$. Structural wingbox mass, including a $25 \%$ knock-up for secondary mass, will vary during optimization between 10,000 and 15,000 $\mathrm{kg}$. Given these values, the takeoff gross weight (TOGW) for the entire vehicle is roughly $300,000 \mathrm{~kg}$. Finally, a subset of the rib-skin intersection nodes are identified as "hard points" for splining to the aerodynamic mesh (seen on the left of Fig. 2) for subsequent aeroelastic computations. The finite plate spline of Ref. 14 is used for this purpose.

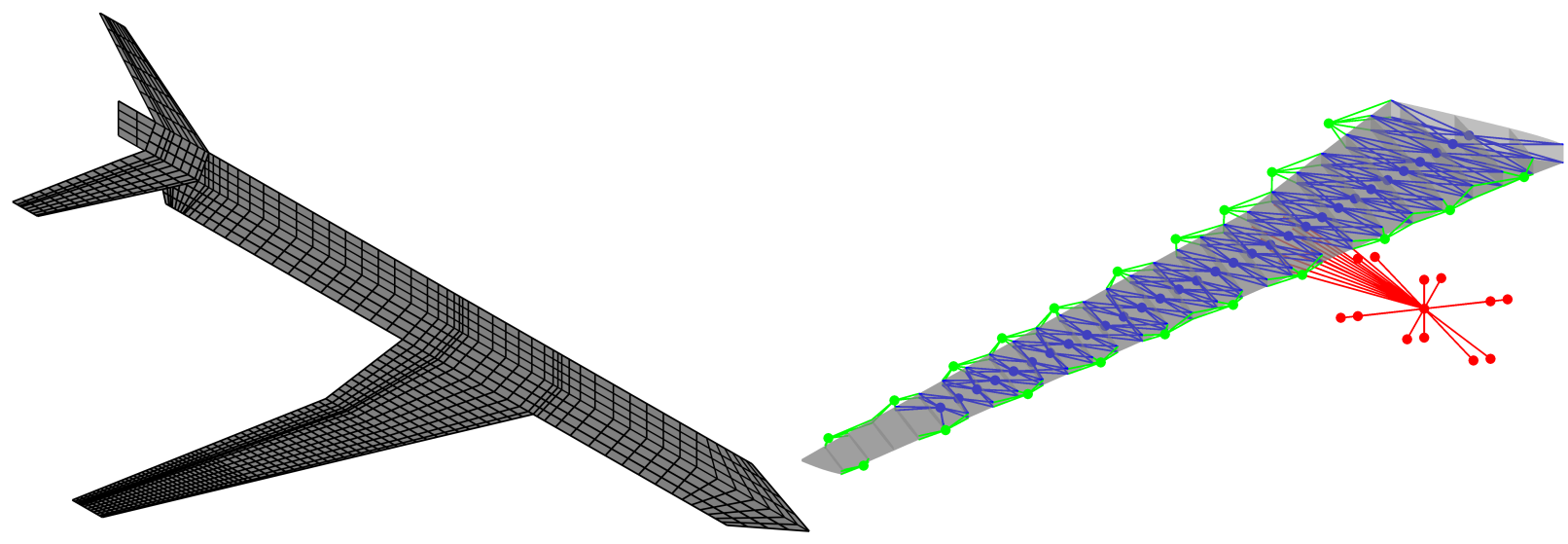

Figure 2. Aerodynamic mesh (left) and distribution of nonstructural lumped mass (right).

\section{B. Aeroelastic Sizing}

Having generated and meshed the wingbox topology, the next step, as noted above, is to freeze this topology and design the sizing details of the structure via aeroelastic optimization. This is done by splitting the upper skin, lower skin, leading spar, trailing spar, and internal ribs each into 5 zones, leaving 25 total. Within each zone, the thickness of each shell member $(t)$, the thickness of the stiffeners attached to these shells $\left(t_{s}\right)$, and the height of the stiffeners $\left(h_{s}\right)$, are independently optimized, leaving 75 total sizing design variables. For this work, the height of each T-stiffener web is set equal to the flange width, and the thickness of the web and flange are equal as well, as drawn in Fig. 3. As noted above, only the skin stiffeners are modeled explicitly; web stiffeners are smeared.

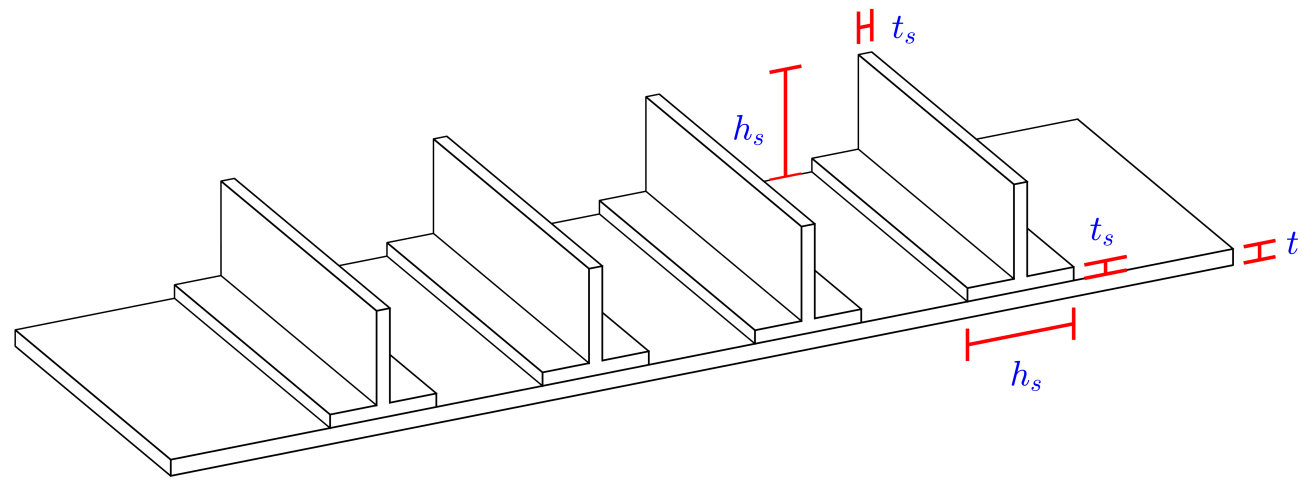

Figure 3. Example T-stiffener geometry.

The wingbox is sized across four maneuver loads, summarized in Table 3. Loads cases 1 and 2 are longitudinal aeroelastic loads, where the angle of attack and tail elevator are used to trim (i.e., set the load 
factor to the proper value, and the pitch rate to zero). Load case 3 is an aeroelastic rolling maneuver at level flight (driven by an outboard aileron), and case 4 is an inertial landing load, without aeroelastic effects. The static aeroelastic analysis tool used here is summarized in Ref. 15. Having computed the trimmed structural deformation for each case, elemental von Mises stresses are computed with an applied safety factor of 1.5, and then compressed into a small number of design constraints via the Kreisselmeier-Steinhauser (KS) function. ${ }^{16}$ All stresses in the upper skins and upper stiffeners are compressed into one $K S$ value, stresses in the lower skins and stiffeners into a second, rib stresses into a third, and spar stresses into a fourth. If each of these $K S$ values is less than zero, then each of the aggregated elemental stresses will lie within their failure envelope.

Finally, the first ten buckling eigenvalues are computed for each load case, and again aggregated into one $K S$ constraint per case. As with the stresses, if each of these $K S$ values is less than zero, then each load case will be free of buckling instabilities. In theory, only the first eigenvalue needs to be contained within the buckling envelope to ensure stability, but the first ten are computed and aggregated into a single $K S$ value, to allow for switching of the critical mode during sizing optimization in a smooth and continuous manner.

Table 3. Summary of static aeroelastic load cases.

\begin{tabular}{rrrrr} 
case & load factor & nondimensional roll rate & Mach & altitude \\
\hline 1 & -1.0 & - & 0.64 & $0 \mathrm{ft}$ \\
2 & 2.5 & - & 0.64 & $0 \mathrm{ft}$ \\
3 & 1.0 & 0.08 & 0.64 & $0 \mathrm{ft}$ \\
4 & 2.0 & - & - & -
\end{tabular}

The final aeroelastic sizing optimization problem is written in Table 4. $\boldsymbol{x}_{\text {sizing }}$ is the vector of design variables: $t, t_{s}$, and $h_{s}$ for each design patch. $t$ and $t_{s}$ are both allowed to range between 3 and $30 \mathrm{~mm}$, while $h_{s}$ ranges between 30 and $100 \mathrm{~mm}$, except in the vertical webs, where the lower bound is $60 \mathrm{~mm}$. $N_{L}$ is the number of load cases, $N_{\sigma}$ is the number of stress-based $K S$ values per load case (four), and $N_{\mu}$ is the number of buckling-based $K S$ values per load case (one). As a surrogate for manufacturing considerations, the shell thickness $t$ must be within $2.5 \mathrm{~mm}$ of the stiffener thickness $t_{s}$, for each design patch. These constraints are aggregated into a single metric, $K S_{t / t_{s}}$. Similarly, the aspect ratio of each stiffener $\left(h_{s} / t_{s}\right)$ should not be greater than 15, which is compressed into a single constraint, $K S_{A R}$. This gradient-based optimization problem is solved with the Globally-Convergent Method of Moving Asymptotes tool (GCMMA) ${ }^{17}$ Design derivatives are analytically computed via the adjoint method. ${ }^{18}$

Table 4. Aeroelastic sizing optimization.

\begin{tabular}{rll} 
minimize: & mass & \\
\hline subject to: & $\boldsymbol{x}_{L B} \leq \boldsymbol{x}_{\text {sizing }} \leq \boldsymbol{x}_{U B}$ & \\
& $K S_{\sigma, i} \leq 0$ & $i=1, \ldots, N_{\sigma} \cdot N_{L}$ \\
& $K S_{\mu, i} \leq 0$ & $i=1, \ldots, N_{\mu} \cdot N_{L}$ \\
& $K S_{t / t_{s}} \leq 0$ & \\
& $K S_{A R} \leq 0$ &
\end{tabular}

\section{Nested Optimization Algorithm}

Each of the topology/layout design variables (10 for straight component designs, 24 for curved) is grouped into the vector $\boldsymbol{x}_{\text {topology }}$, and each of the sizing design variable $\left(75\right.$ total) is grouped into $\boldsymbol{x}_{\text {sizing }}$. The nested optimization algorithm used to simultaneously handle the topology/layout design variables (Table 1 or Table 2) and the sizing design variables (Fig. 3) is given as:

1. Generate an initial population of $\boldsymbol{x}_{\text {topology }}$ designs using Latin hypercube sampling (LHS).

2. For each topological design, solve the problem in Table 4 to optimize the corresponding sizing design variables $\boldsymbol{x}_{\text {sizing }}$. Return the objective function (mass) for each design. The sizing constraints $\left(K S_{\sigma}\right.$, 
etc.) will be satisfied (i.e., set to 0 for active constraints) by the GCMMA algorithm, and do not need to be returned.

3. Fit the mass function with a radial basis function (RBF) surrogate model across the $\boldsymbol{x}_{\text {topology }}$ design space.

4. Use the surrogate model to find an in-fill point: i.e., the next topological design worth exploring.

5. Solve the sizing optimization problem (Table 4) for the new $\boldsymbol{x}_{\text {topology }}$ in-fill point, and then recompute the surrogate model with this new data point included.

6. Return to step 4, and repeat until some convergence criteria is attained.

The in-fill strategy used here is based on the MISO (Mixed Integer Surrogate Optimization) CPTV algorithm described in Ref. 19, which is a combination of coordinate perturbation (CP) and target value (TV) strategies. CP involves randomly varying the members of the current-best $\boldsymbol{x}_{\text {topology }}$ design subject to some specified standard deviation $\sigma$. The in-fill point is found which, of these random designs, minimizes a weighted combination of a distance metric (proximity to the current-best $\boldsymbol{x}_{\text {topology }}$ ) and the objective function as predicted by the RBF. TV involves minimizing a "bumpiness measure" pursuant to a targeted improvement that is some percentage beyond the current best performance (mass, in this case). The original CPTV algorithm detailed in that paper involves cycling through various $\mathrm{CP}$ and TV strategies from one in-fill cycle to the next, depending on how well the previous in-fill point performs when analyzed/optimized in Step 5, with patterned changes in $\sigma$ and the distance/performance weighting value in CP, and the targeted improvement in TV. Additional details can be found in Ref. 19.

In this work, rather than computing a single in-fill $\boldsymbol{x}_{\text {topology }}$ point per iteration in steps 4 and 5 , fifteen in-fill $\boldsymbol{x}_{\text {topology }}$ points are computed, and each one aeroelastically sized via Table 4 at once, in parallel. Rather than cycling through various CP/TV strategies in serial, as done in Ref. 19, each of the various strategies are implemented at once: nine CP in-fill points (a 3-by-3 array of $\sigma$ values and distance/performance weighting values) and six TV in-fill points (six different targeted improvement thresholds, ranging from a moderate $0.01 \%$ targeted improvement, to an ambitious $10 \%$ improvement in the objective function). Having computed the optimal structural mass for all 15 in-fill points, these points are all added to the database of $\boldsymbol{x}_{\text {topology }}$ designs, the $\mathrm{RBF}$ is recomputed in step 5 , and the process repeats.

Computing multiple in-fill points in parallel is a known strategy for insuring against poor-performing data points ${ }^{20}$ (i.e., an in-fill point that the RBF thinks is a worthwhile design, but turns out not to be once the optimization problem in Table 4 is actually solved), a particularly important tactic for the larger topological design spaces considered here.

\section{Results: Straight Components}

The first set of results shown here will be restricted to straight components (i.e., ribs and stiffeners) only. For this 10-design variable case, an initial $\boldsymbol{x}_{\text {topology }}$ population of 240 designs is derived via LHS. The optimal structural mass (objective function) and the sizing constraint violation of each design is shown in Fig. 4, before and after the aeroelastic sizing optimization process. The sizing optimization history of four of these designs is shown in Fig. 5. Most of these 240 designs are infeasible prior to sizing optimization (i.e., either buckles or experiences stress-based failure within the design envelope), and require sizing to bring this constraint violation to zero, which in turn cause the structural mass to rise. This reinforces an idea mentioned above, that topology design variables, on their own, will struggle to produce a feasible wingbox design. One exception to this is design 176, whose presized design is feasible, and for whom aeroelastic sizing actually reduces structural mass. The final structural mass of this design, $12,690 \mathrm{~kg}$, is the lightest of the set in Fig. 4.

Using this initial set of 240 designs, the MISO-CPTV process itemized above is implemented: convergence is shown in Fig. 6. The initial structural mass is $12,690 \mathrm{~kg}$, which is the best design found among the initial LHS population, and converges to a structural mass of $12,258 \mathrm{~kg}$ after 55 iterations. It is re-emphasized here that each data point in this plot represents a complete gradient-based sizing optimization of 15 in-fill points at once; the structural mass reported in Fig. 6 is the lowest value found up to that point. For some of the topology optimization iterations in Fig. 6, the structural mass does not decrease (iterations 5 and 6 , for example). In each of these cases, none of the 15 in-fill points is any better than the existing database of designs, despite the RBF surrogate model's hypothesis otherwise. The RBF model is then recomputed with these 15 poor-performing data points, and the prediction error in this region is decreased. 

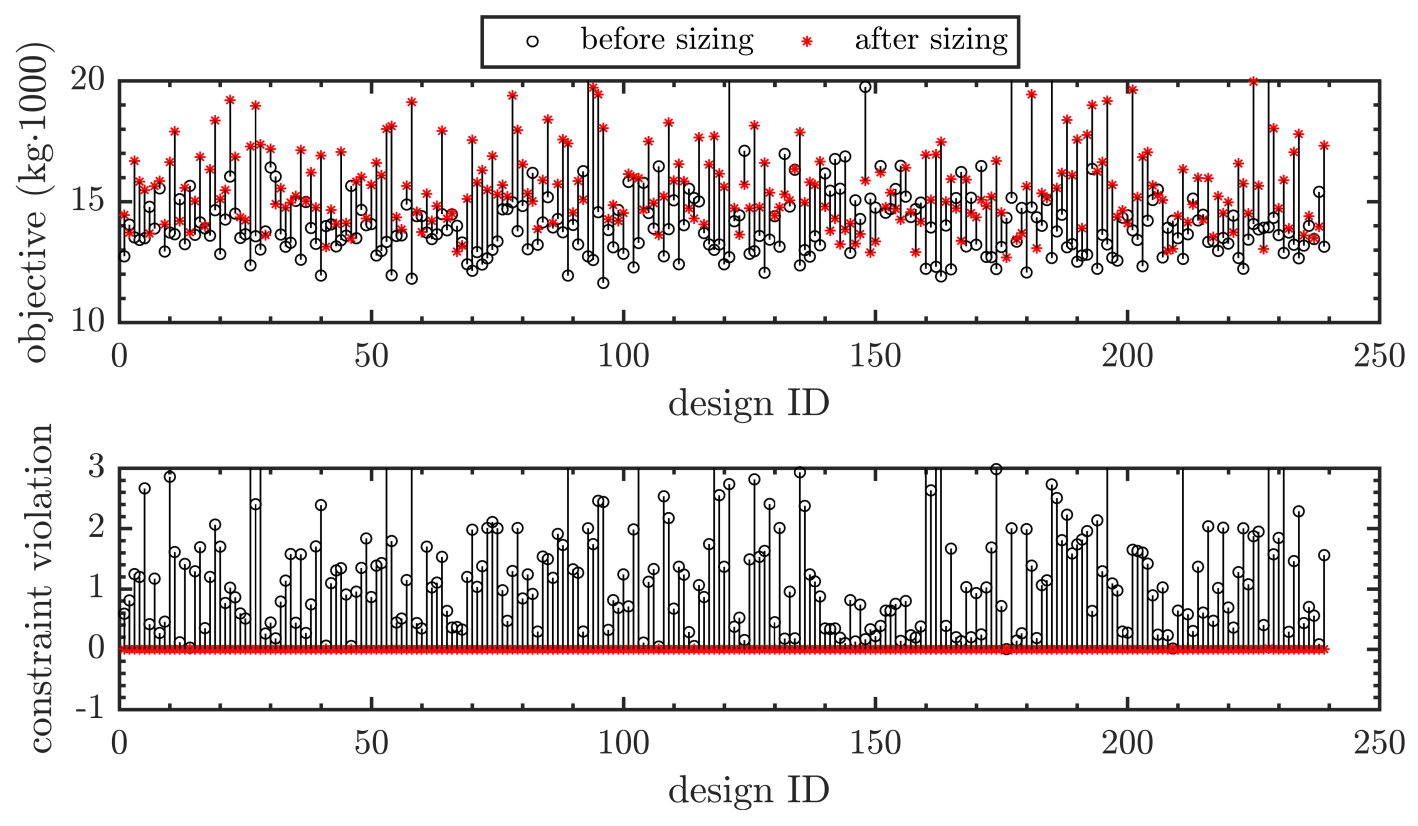

Figure 4. Initial population of straight-member topologies, before and after sizing optimization.
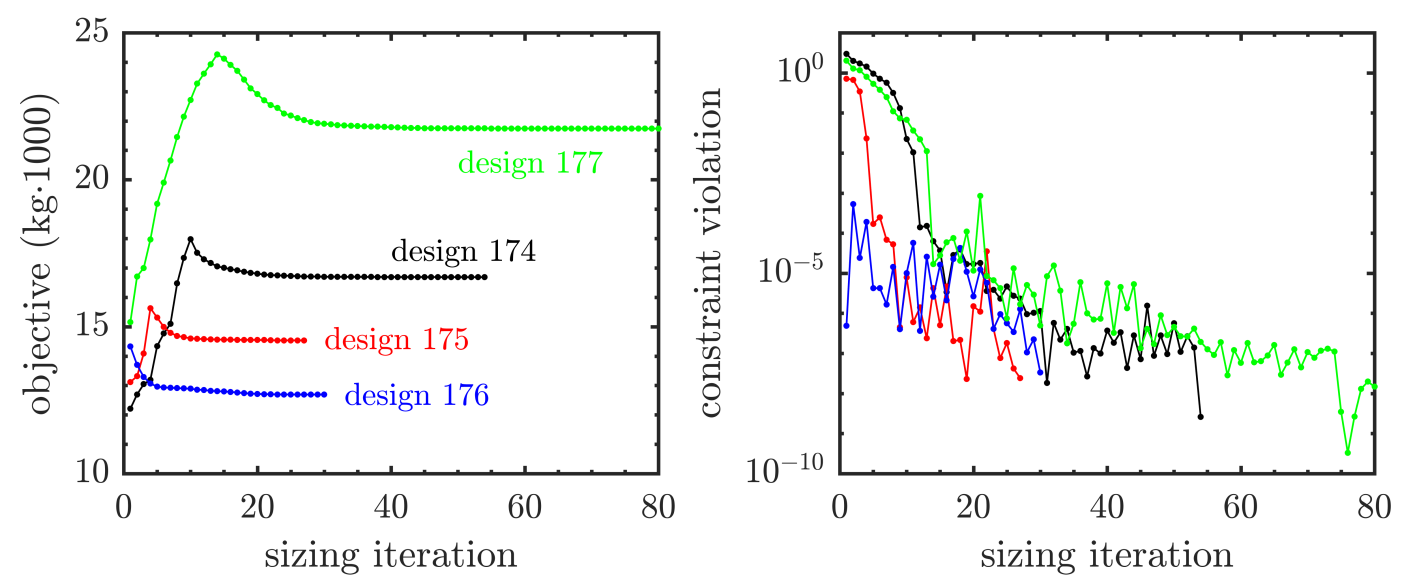

Figure 5. Gradient-based optimization of designs 174-177 from Fig. 4.

As noted in the Introduction, the typical optimizer used with surrogate-based design is an evolutionary optimizer. For comparison purposes, the convergence of a genetic algorithm (GA) is also shown in Fig. 6. MISO-CPTV finds a better design in far fewer iterations (a result also found in Ref. 19), though no effort is made here to explore the best GA settings for a complex problem of this nature. Namely, a population of size of 100 is used along with an elitism count of 5 , but only the best 20 designs at each generation are selected for gradient-based optimization, in order to reduce the computational cost. The resulting "hybrid" GA (i.e., one that combines the evolutionary operators with a gradient-based search) is well-studied in the literature, ${ }^{21}$ though most of the existing studies consider the same set of design variables for both the evolutionary step and the gradient-based step. In this work, of course, two different sets of design variables are considered: $\boldsymbol{x}_{\text {topology }}$ and $\boldsymbol{x}_{\text {sizing. }}$.

The convergence of the various design variables in Table 1 for the MISO-CPTV process is also shown in Fig. 6 (GA parameter convergence is not shown), with the optimizer eventually settling on 27 upper skin stiffeners and 12 lower skin stiffeners. This is driven by the fact that the buckling requirements are 
less stringent for the lower skins than the upper skins, as the negative load factors in Table 3 are not as aggressive as the positive factors. Both sets of stiffeners are rotated forward relative to the leading spar (a known strategy for passive load alleviation ${ }^{15,22}$ ), with lower pitch toward the trailing edge compared to the leading edge. The stiffener pitch discrepancy is likely driven by the trailing edge break in the wingbox planform, which will lead to stress concentrations. For similar reasons, the rib pitch at the break is lower than found at the wing root. It is finally noted that the component spacing plot of Fig. 6 has been nonlinearly scaled from the $0.25-4.0$ bounds noted in Table 1 to $0-1$ bounds, for plotting purposes only.
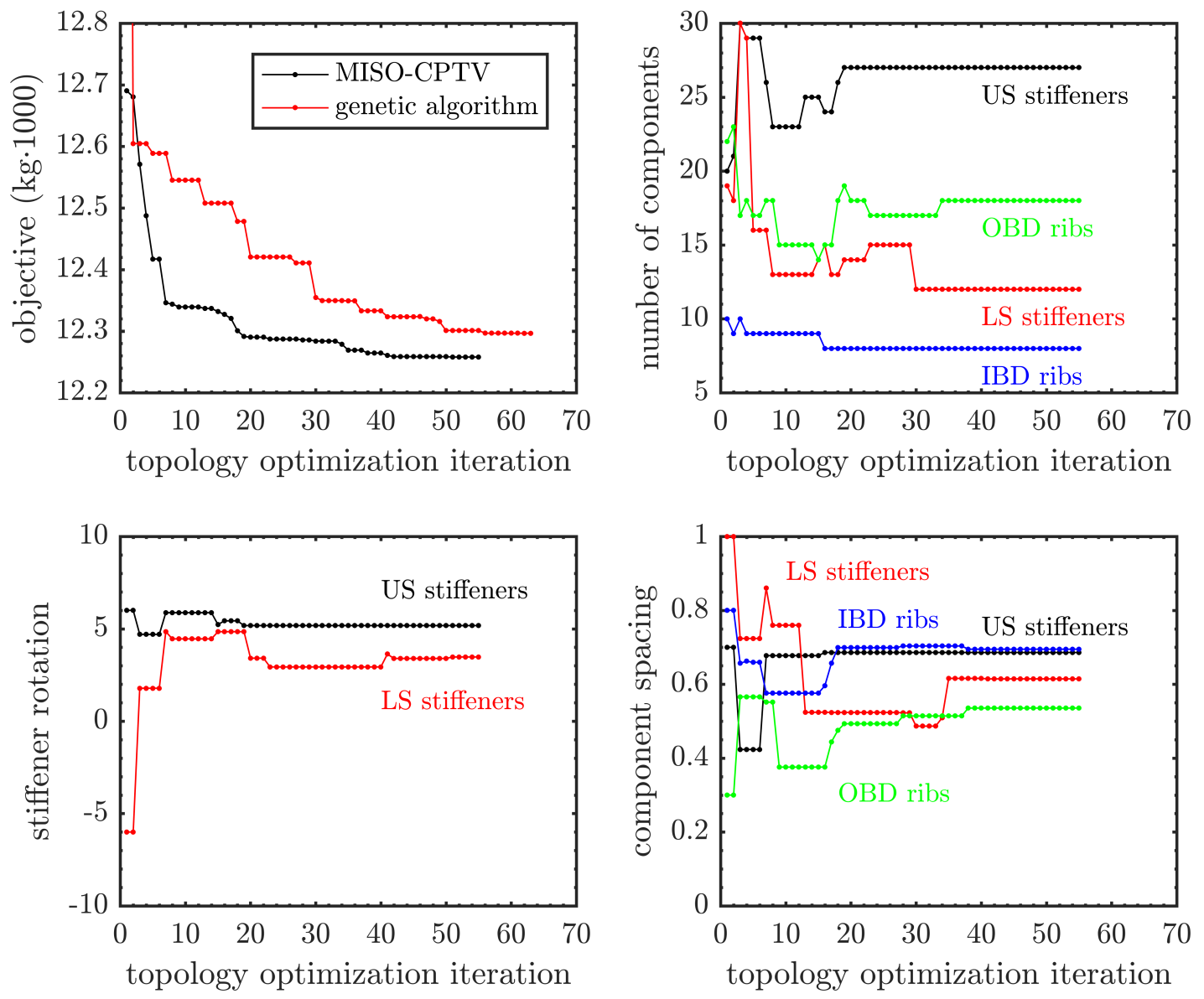

Figure 6. Convergence of the MISO-CPTV process for the straight-component case. "IBD" and "OBD" stand for "inboard" and "outboard", "US" and "LS" stand for "lower skin" and "upper skin".

The optimal sizing distribution of the final result is shown in Fig. 7, in terms of $t, t_{s}$, and $h_{s}$. The majority of the aluminum material is allocated to the wing skins, with thicker shells (covers) in the lower skins, but taller stiffeners in the upper skins. The sizing parameters of the ribs and spars are largely pushed to their lower bounds, which may be partially due to the fact that crushing loads are not included here. The aeroelastic behavior of this optimal wingbox is summarized in Figures 8, 9, and 10, which document the static aeroelastic deflections, the resulting stress distributions, and the critical buckling eigendata for each load case in Table 3. The stress constraint is only active for load cases 2 and 3, with large stresses in the upper and lower skins, rear spar, and (for the rolling maneuver case 3) the leading spar as well. Normalized stresses in these locations in Fig. 9 appear slightly less than unity owing to the conservatism of the $K S$ approach.

The buckling constraint, on the other hand, is active for all four load cases in Fig. 10, though again the critical eigenvalue reported in the figure is less than one owing to the conservatism of the constraint aggregation. Eigenvalues much lower than one (load case 3, for example) are indicative of a situation where the subcritical buckling modes are very aggressive, inducing greater conservatism in the $K S$ method. ${ }^{16}$ The 
critical buckling eigenmodes of load cases 2, 3, and 4 are characterized by intrapanel deformation along adjacent rib bays, due in part to the fact that the rib webs are not thick enough to force a local buckling mechanism. Load case 1, however, is characterized by a local buckling mode in the outboard panels.

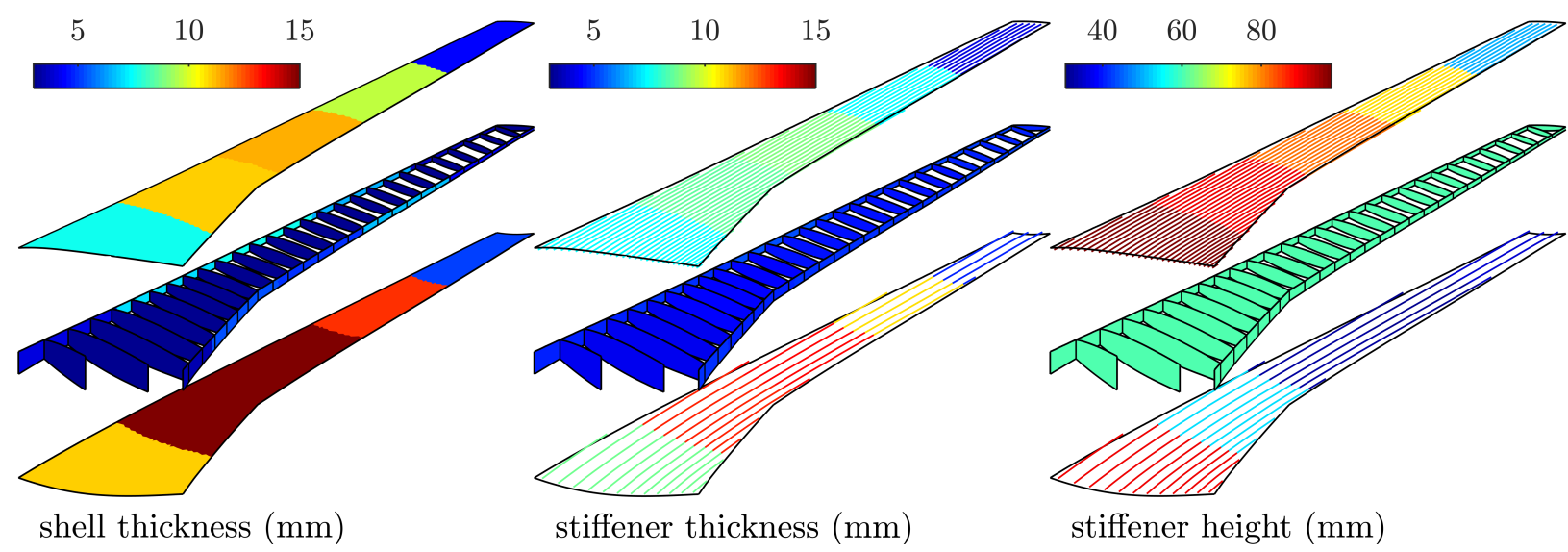

Figure 7. Final sizing variables for the straight-component case.

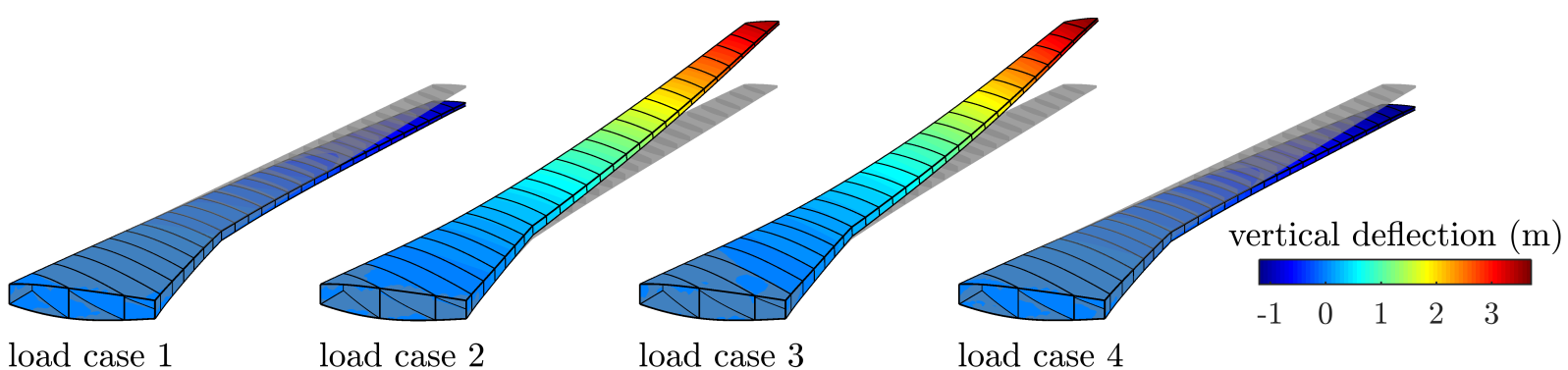

Figure 8. Aeroelastic deflections for the optimal wingbox with straight components.

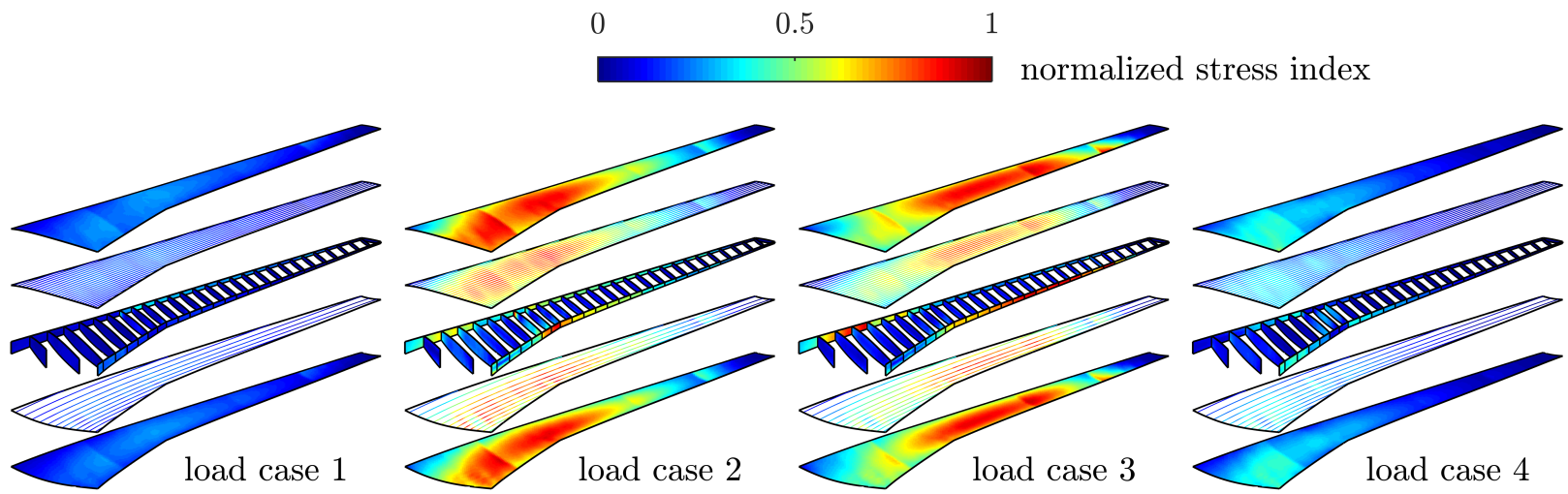

Figure 9. Stress distribution for the optimal wingbox with straight components.

\section{Results: Curved Components}

Having solved the wingbox optimization problem with straight components (Table 1), the process is repeated for curvilinear components (Table 2), a harder problem to solve with 24 topological design variables, rather than 10. An initial database of 5,000 sized LHS designs is used to start the process, similar to data shown in Fig. 4 for the straight member case. Of these designs, the minimum structural mass is 12,584 kg. The subsequent convergence of the MISO-CPTV algorithm is shown in Fig. 11, where each iteration again 


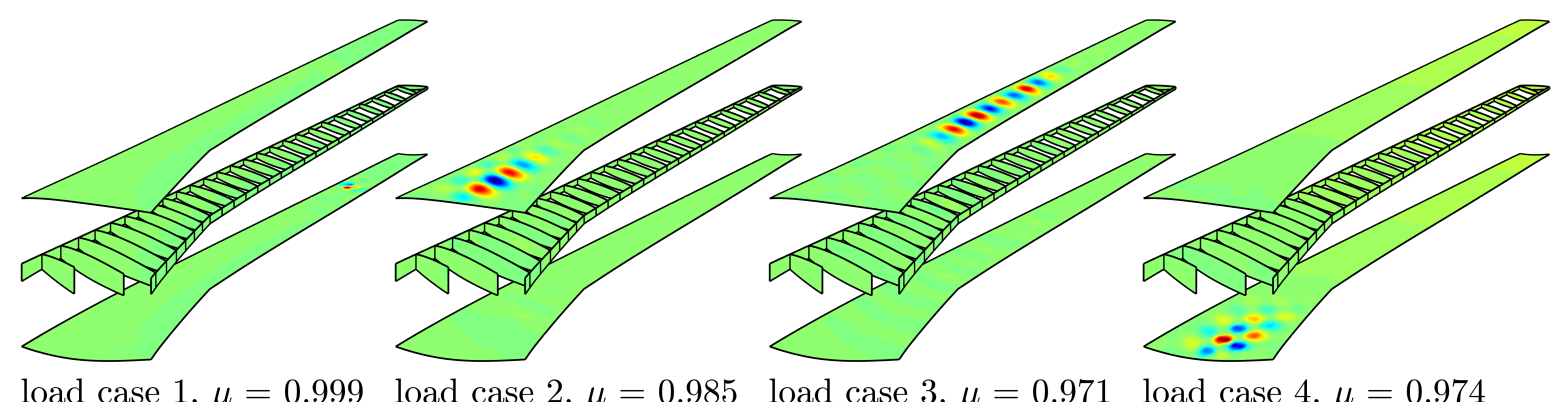

Figure 10. Critical buckling modes for the optimal wingbox with straight components.

involves a complete gradient-based sizing optimization of 15 in-fill data points, in parallel. Structural mass, after 120 iterations, decreases to $12,114 \mathrm{~kg}$, which is $144 \mathrm{~kg}$ lighter than the best design found with straight components only: a $1.18 \%$ decrease.

The optimal topology is shown in Fig. 12, for comparison with the optimal layout restricted to straight components. The inboard ribs are largely straight; some curvature is noted in the outboard rib section, but only in the ribs near the trailing edge break. The ribs at the wingtip are also straight. Stiffener curvature is more pronounced in the lower skins than the upper skins, with an inflection point in the stiffener curve near the break location. This is a clear attempt by the optimizer to attenuate the stress concentrations induced by the break in the geometry.

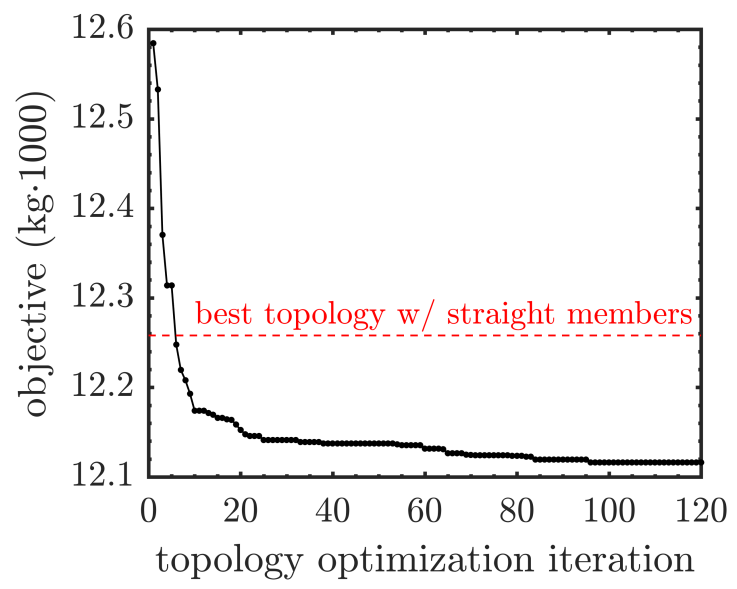

Figure 11. Convergence of the MISO-CPTV process for the curved-component case.

The component mass down the wingspan, for each of the five spanwise design zones indicated in Fig. 7, can be seen in Fig. 13 for the optimal designs with straight and curved members. In keeping with the $1.18 \%$ mass difference between the two designs, the differences in these plots are moderate. A uniform mass decrease in the upper skins is noted between the straight and curved designs, though this is partially offset by heavier upper skin stiffeners in the curved design. The rib mass changes in a nonmonotonic manner from one zone to the next, but all other component masses are largely unchanged. It should be noted that some of these component masses will be impacted by changes in the sizing design variables $\boldsymbol{x}_{\text {sizing }}$ only (the wing skins, for example), while other components will be impacted by changes in both topology and sizing (ribs and skin stiffeners).

At the conclusion of the convergence in Fig. 11, the RBF surrogate model is based upon 6,800 designs: the initial 5,000 LHS designs, and the subsequent 1,800 designs computed as in-fill points. This surrogate model can be used to compute a global sensitivity analysis, to understand which of the $24 \boldsymbol{x}_{\text {topology }}$ design variables has the greatest impact on the optimal structural wingbox mass. Global sensitivities are computed with the Fourier Amplitude Sensitivity Test (FAST) method, using the GSAT toolbox, ${ }^{23}$ and are shown in Fig. 14. Two cases are shown in this figure: the first follows every other result in this paper, where each 


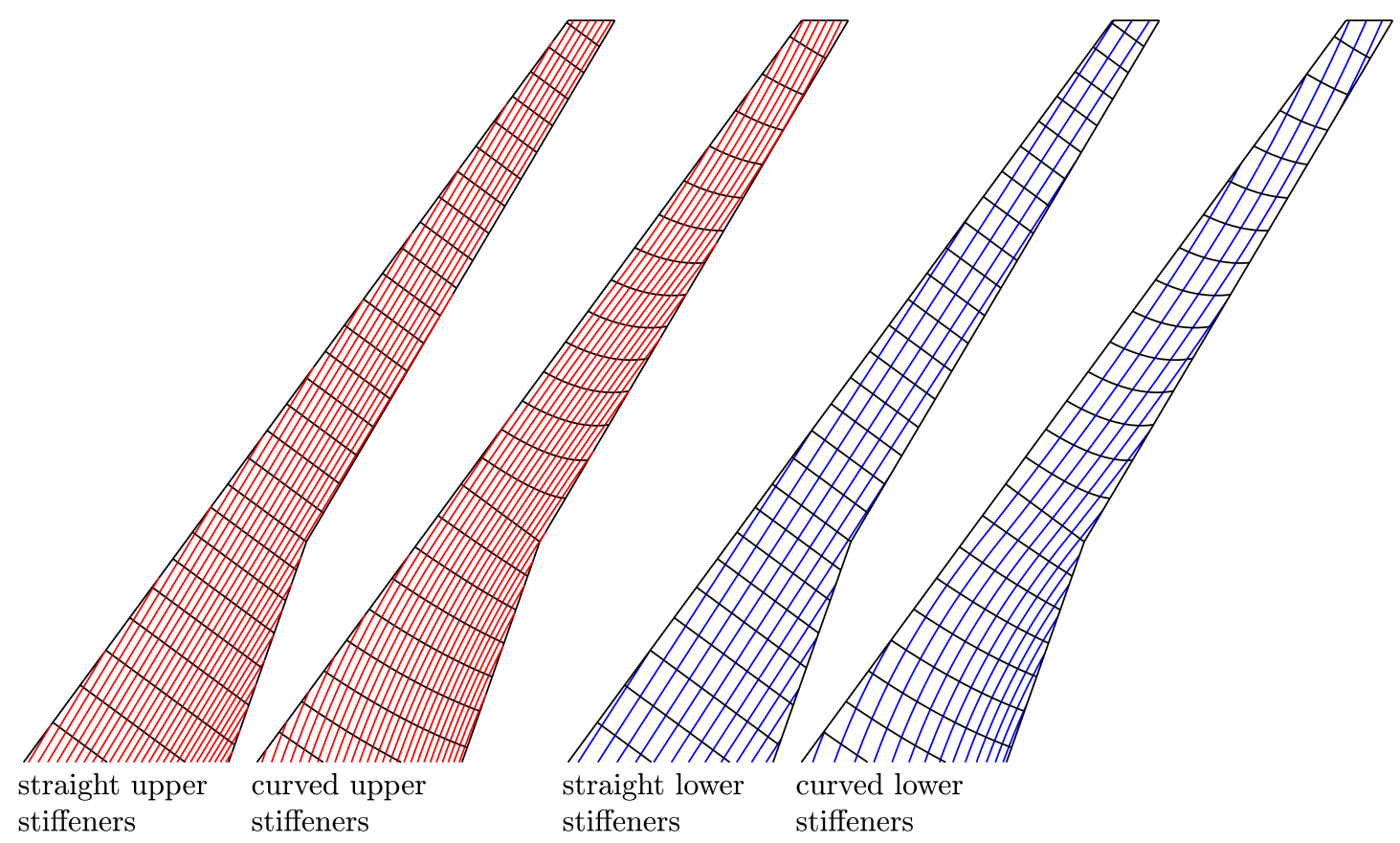

Figure 12. Comparison of straight and curved rib/stiffener layouts.

change to the wing layout $\left(\boldsymbol{x}_{\text {topology }}\right)$ triggers a complete gradient-based sizing optimization of $\boldsymbol{x}_{\text {sizing }}$. As such, the global sensitivities of the structural mass in the top plot of Fig. 14 reflect this dependency.

The bottom plot of Fig. 14 is far simpler, in that $\boldsymbol{x}_{\text {sizing }}$ is frozen across the topological design space. Sizing optimization is not included here, and so the structural mass is not optimal in the sense that aeroelastic constraints will not typically be satisfied. These two plots in Fig. 14 may be thought of as "partial" and "total" structural mass sensitivities with respect to the topological layout. The "partial" sensitivities capture the structural mass increase when the number of ribs is increased (for example), whereas the "total" sensitivities capture the mass change (either increase or decrease) when all of the wingbox components are resized to ensure satisfaction of all aeroelastic constraints, in response to that increase in the number of ribs.

Focusing first on the top plot of global sensitivities in Fig. 14 ("total" sensitivities), it can be seen that the number of inboard ribs is the dominant factor. This is presumably due to the buckling constraints of load cases 2 and 4, where the spatial placement of the buckling lobes are clearly driven by the rib pitch in Fig. 10. The second-ranking global sensitivity is the number of upper skin stiffeners (again driven by buckling concerns), followed by the upper skin stiffener rotation at $2 \cdot y / b=0.29$ (whose importance has been previously noted in Fig. 12, where the inflection point of the curvilinear path is tuned to coincide with the break in the trailing edge). The layout details of the lower skin stiffeners are relatively unimportant, with the exception of the inboard curvilinear control points, and the outboard rib details are relatively unimportant as well. This latter fact is surprising in light of the buckling patterns observed for load case 3 in Fig. 10, which are clearly driven by the rib pitch, and would suggest that this constraint, though active, is not as strong a driver as the buckling of the other cases. It is finally noted that some of the parameters used to precisely control the rib curvature details $\left(\eta_{1}\right.$ and $\eta_{2}$, for example) register with near-zero sensitivities in Fig. 14, suggesting that this level of shape control is overkill for the current problem.

The "partial" sensitivities in the lower portion of Fig. 14, on the other hand, are entirely different, reinforcing the strong role played by the gradient-based sizing optimization previously explored in Fig. 4. For this simpler case, the number of upper and lower skin stiffeners have the biggest impact on the unsized nonoptimal wingbox mass, due to the shear acreage of these structures. The impact of these stiffener components is blunted when sizing optimization is included, as increasing (for example) the number of stiffeners simply allows the gradient-based optimizer to thin-out the skins, a trade-off noted in Fig. 13. The opposite trend is observed with the number of inboard ribs, which has a relatively small "partial" sensitivity (i.e., relatively low acreage), but becomes the dominant factor when aeroelastic resizing is included. 

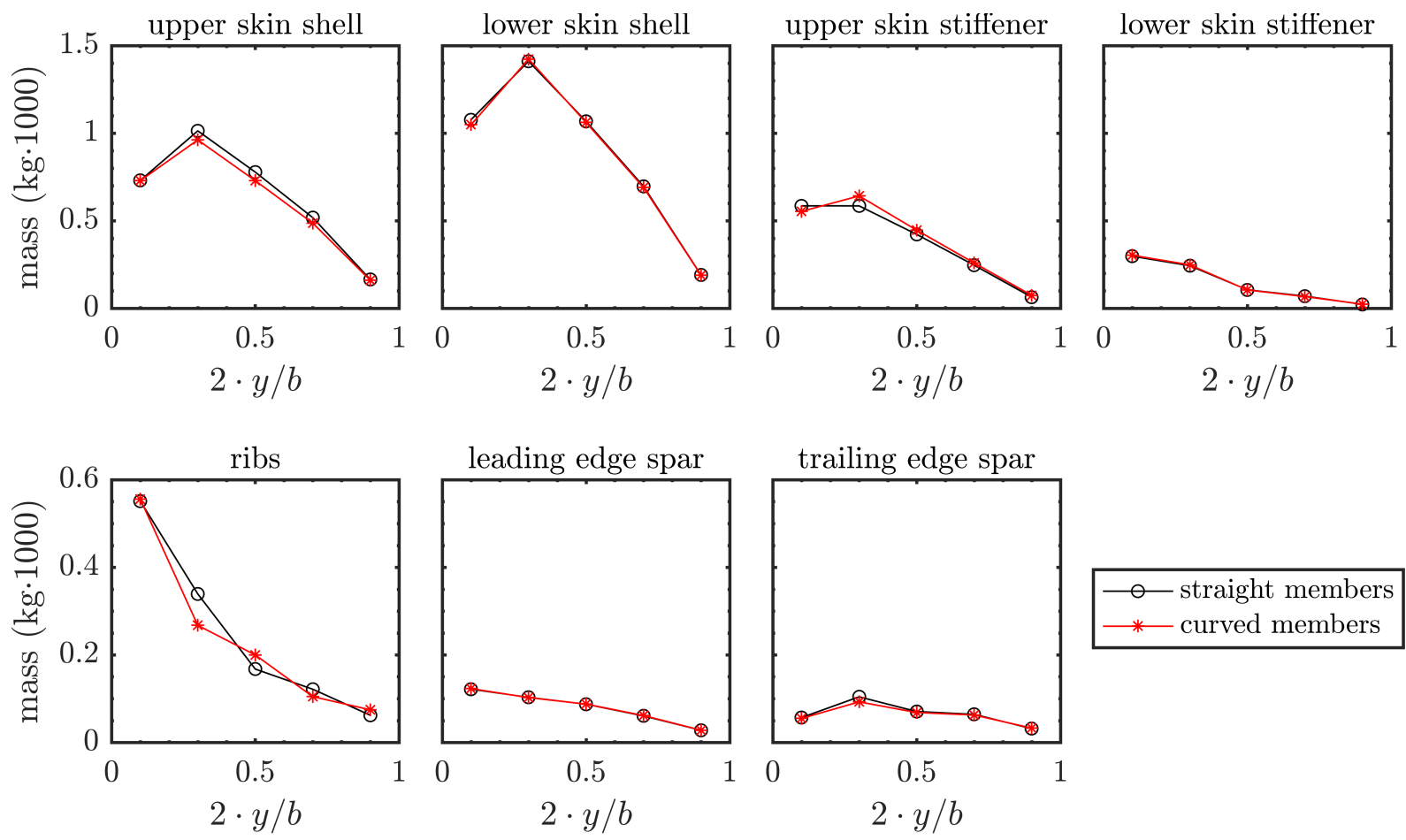

Figure 13. Optimal component mass down the wingspan.

\section{Conclusions}

A nested optimization procedure has been utilized here in order to simultaneously handle wingbox rib and skin stiffener layout design variables, as well as a spatially-detailed set of component sizing design variables. A version of the mixed integer surrogate in-fill optimizer, MISO-CPTV, is used to optimize the topology design variables. Each wingbox topology that comes up for consideration by MISO-CPTV is subjected to a gradient-based sizing optimization of all components. The resulting algorithm, though computationally expensive, is demonstrated to outperform competing algorithms that have been used in the literature (typically based on evolutionary operators). Two optimizations are performed here: one that is limited to straight ribs and stiffeners only (10 topological design variables) and one that allows these members to become curved ( 24 design variables). The number of sizing variables that are optimized at the lower level of the nested procedure is 75 for both cases, and details the shell thickness, stiffener thickness, and stiffener height of several sizing zones along the wingbox. The structural mass reduction afforded by the use of curvilinear members is only moderate: a $1.18 \%$ decrease.

Future work may expand the scope here to include design changes to the jig shape outer mold line (which is frozen here), and move from a structural weight reduction objective function, to one that accounts also for fuel burn. It will be of interest to understand the way in which the optimal rib/stiffener topology changes in response to the span extension needed to accommodate lower-drag designs.

\section{References}

\footnotetext{
${ }^{1}$ Balabanov, V., Haftka, R., "Topology Optimization of Transport Wing Internal Structure," Journal of Aircraft, Vol. 33, No. 1, pp. 232-233, 1996.

${ }^{2}$ Locatelli, D., Mulani, S., Kapania, R., "Wing-Box Weight Optimization Using Curvilinear Spars and Ribs (SpaRibs)," Journal of Aircraft, Vol. 48, No. 5, pp. 1671-1684, 2011.

${ }^{3}$ Wang, W., Guo, S., Yang, W., "Simultaneous Partial Topology and Size Optimization of a Wing Structure Using Ant Colony and Gradient Based Methods," Engineering Optimization, Vol. 43, No. 4, pp. 433-446, 2011.

${ }^{4}$ Kolonay, R., Kobayashi, M., "Optimization of Aircraft Lifting Surfaces using a Cellular Division Method," Journal of Aircraft, Vol. 52, No. 6, pp. 2051-1684, 2015.
} 


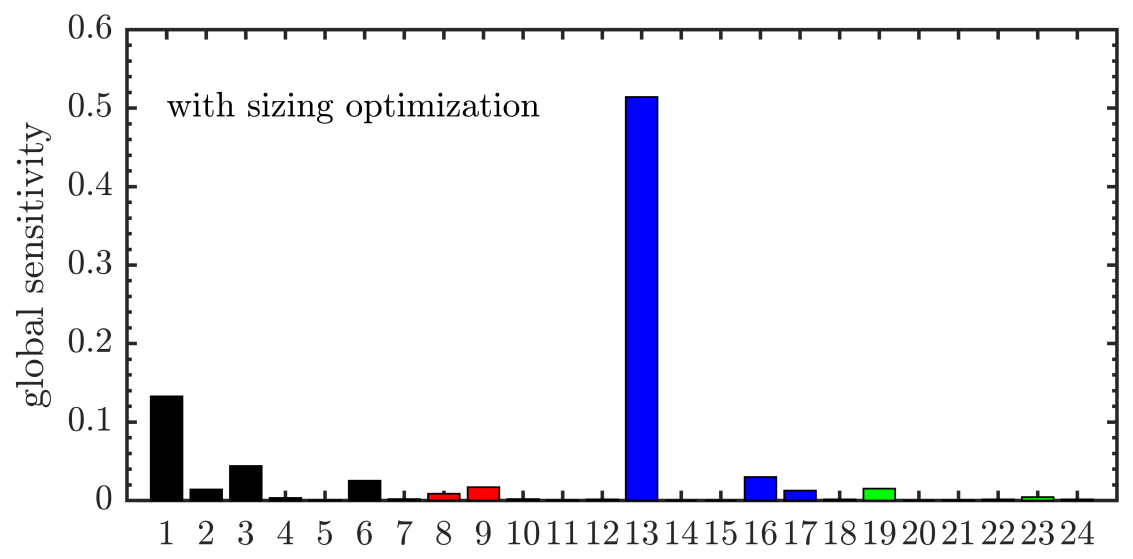

1: \# of upper skin stiffeners

2: upper skin stiffener rotation 1

3: upper skin stiffener rotation 2

4: upper skin stiffener rotation 3

5: upper skin stiffener rotation 4

6: upper skin stiffener spacing

7: \# of lower skin stiffeners

8: lower skin stiffener rotation 1

9: lower skin stiffener rotation 2

10: lower skin stiffener rotation 3

11: lower skin stiffener rotation 4

12: lower skin stiffener spacing

13: \# of inboard ribs

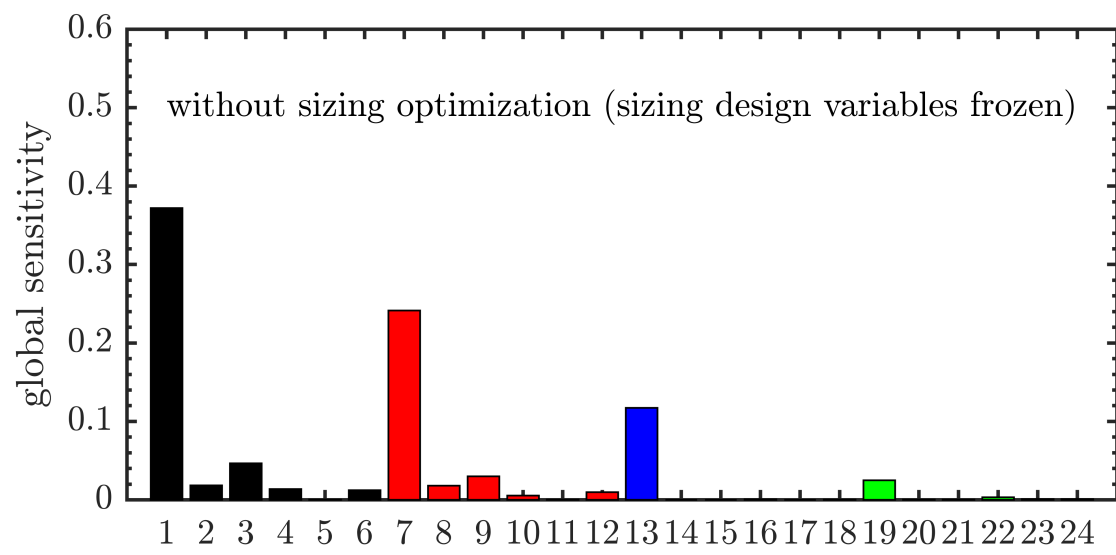

14: inboard rib $\eta_{1}$

15: inboard rib $\eta_{2}$

16: inboard rib $\xi_{12} / \xi_{11}$

17: inboard rib $\xi_{22} / \xi_{21}$

18: inboard rib $\xi_{32} / \xi_{31}$

19 \# of outboard ribs

20: outboard rib $\eta_{1}$

21: outboard rib $\eta_{2}$

22: outboard rib $\xi_{12} / \xi_{11}$

23: outboard rib $\xi_{22} / \xi_{21}$

24: outboard rib $\xi_{32} / \xi_{31}$

Figure 14. Global sensitivity of the structural mass with respect to the topological design variables.

${ }^{5}$ Dubois, A., Farhat, C., Abukhwejah, A., "Parameterization Framework for Aeroelastic Design Optimization of BioInspired Wing Structural Layouts," AIAA Structures, Structural Dynamics, and Materials Conference, San Diego, CA, January $4-8,2016$.

${ }^{6} \mathrm{Jrad}$, M., De, S., Kapania, R., "Global-Local Aeroelastic Optimization of Internal Structure of Transport Wing," AIAA Multidisciplinary Analysis and Optimization Conference, Denver, CO, June 5-9, 2017.

${ }^{7}$ Locatelli, D., private communication, 2016.

${ }^{8}$ Hansen, L., Horst, P., "Multilevel Optimization in Aircraft Structural Design Evaluation," Computers and Structures, Vol. 86, No. 1, pp. 104-118, 2008.

${ }^{9}$ Roy, S., Moore, K., Hwang, J., Gray, J., Crossley, W., Martins, J., "A Mixed Integer Efficient Global Optimization Algorithm for the Simultaneous Aircraft Allocation-Mission-Design Problem," AIAA Structures, Structural Dynamics, and Materials Conference, Grapevine, TX, January 9-13, 2017.

${ }^{10}$ Vassberg, J., DeHaan, M., Rivers, S., Wahls, R., "Development of a Common Research Model for Applied CFD Validation Studies," AIAA Applied Aerodynamics Conference, Honolulu, Hawaii, August 10-13, 2008.

${ }^{11}$ Kenway, G., Martins, J., Kennedy, G., "Aerostructural Optimization of the Common Research Model Configuration," AIAA Aviation Conference, Atlanta, GA, June 16-20, 2014.

${ }^{12}$ Stroud, W., Agranoff, N., "Minimum-Mass Design of Filamentary Composite Panels under Combined Loads: Design Procedure Based on Simplified Buckling Equations," NASA TN D-8257, 1976.

${ }^{13}$ Hwang, J., Martins, J., "An Unstructured Quadrilateral Mesh Generation Algorithm for Aircraft Structures," Aerospace Science and Technology, Vol. 59, No. 1, pp. 172-182, 2016.

${ }^{14}$ Appa, K., "Finite-Surface Spline," Journal of Aircraft, Vol. 26, No. 5, pp. 495-496, 1989.

${ }^{15}$ Stanford, B., Jutte, C., "Comparison of Curvilinear Stiffeners and Tow Steered Composites for Aeroelastic Tailoring of Transports," Computers and Structures, Vol. 183, No. 1, pp. 48-60, 2017.

${ }^{16}$ Kreisselmeier, G., Steinhauser, R., "Systematic Control Design by Optimizing a Vector Performance Index", International Federation of Active Controls Symposium on Computer-Aided Design of Control Systems, Zurich, Switzerland, 1979.

${ }^{17}$ Svanberg, K., "A Class of Globally Convergent Optimization Methods Based on Conservative Convex Separable Approximations," SIAM Journal of Optimization, Vol. 12, pp. 555-573, 2002.

${ }^{18}$ Adelman, H., Haftka, R., "Sensitivity Analysis of Discrete Structural Systems," AIAA Journal, Vol. 24, No. 5, pp. 823-832, 1986. 
${ }^{19}$ Müller, J., "MISO: Mixed Integer Surrogate Optimization Framework," Optimization and Engineering, Vol. 17, No. 1, pp. 177-203, 2016.

${ }^{20}$ Haftka, R., Villanueva, D., Chaudhuri, A., "Parallel Surrogate-Assisted Global Optimization with Expensive Functions -a Survey," Structural and Multidisciplinary Optimization, Vol. 54, No. 1, pp. 3-13, 2016.

${ }^{21}$ El-Mihoub, T., Hopgood, A., Nolle, L., Battersby, A., "Hybrid Genetic Algorithms: a Review," Engineering Letters, Vol. 13, No. 1, pp. 124-137, 2006.

${ }^{22}$ Williams, J., "Analysis/Theory of Controlled Configured Structures," AFFDL-TR-74-137, Air Force Flight Dynamics Laboratory, 1974.

${ }^{23}$ Cannavó, F., "Sensitivity Analysis for Volcanic Source Modeling Quality Assessment and Model Selection" Computers and Geosciences, Vol. 44, No. 1, pp. 52-59, 2012. 